\title{
Photon Temporal Modes: A Complete Framework for Quantum Information Science
}

\author{
B. Brecht, ${ }^{1}$ Dileep V. Reddy, ${ }^{2}$ C. Silberhorn, ${ }^{1}$ and M. G. Raymer ${ }^{2}$ \\ ${ }^{1}$ Integrated Quantum Optics, Applied Physics, University of Paderborn, \\ Warburger Strasse 100 33098, Paderborn, Germany \\ ${ }^{2}$ Department of Physics, Oregon Center for Optics, University of Oregon, Eugene, Oregon 97403, USA \\ (Received 23 April 2015; revised manuscript received 21 July 2015; published 30 October 2015; corrected 21 January 2016)

\begin{abstract}
Field-orthogonal temporal modes of photonic quantum states provide a new framework for quantum
\end{abstract} \\ information science (QIS). They intrinsically span a high-dimensional Hilbert space and lend themselves to \\ integration into existing single-mode fiber communication networks. We show that the three main \\ requirements to construct a valid framework for QIS - the controlled generation of resource states, the \\ targeted and highly efficient manipulation of temporal modes, and their efficient detection-can be fulfilled \\ with current technology. We suggest implementations of diverse QIS applications based on this complete \\ set of building blocks.
}

DOI: 10.1103/PhysRevX.5.041017

\section{INTRODUCTION}

Quantum information science (QIS) offers means for storing, transmitting, and processing information in ways not achievable using classical information technology. Examples of the benefits of QIS are unconditionally secure communication, ultraprecise metrology beyond classical limits, and superior computational algorithms.

While all of those can theoretically be realized using only photons, it is generally accepted that quantum computation will be implemented in material systems, whereas quantum communication (QC) and information transfer across a distributed quantum network-a so-called "quantum internet" [1]—will be based on photons. Strongly interacting material systems, which can be controlled with outstanding precision, facilitate the implementation of stationary logical processors and quantum memories. The latter are an indispensable building block for longdistance entanglement distribution via quantum repeaters, which in turn is inextricably linked with secure longdistance quantum communication. Photons, in contrast, interact only weakly with themselves and their environment, meaning that they experience very low decoherence. Thus, they are naturally suited for carrying fragile quantum information over transmission lines in a network. The remaining challenge for these hybrid network architectures is the efficient interfacing of flying qubits (photons) and stationary qubits (material systems), which is complicated by the fact that most practical material systems have stringent requirements on the photon spectral-temporal

Published by the American Physical Society under the terms of the Creative Commons Attribution 3.0 License. Further distribution of this work must maintain attribution to the author(s) and the published article's title, journal citation, and DOI.
Subject Areas: Quantum Information

amplitude. Thus, small photonic coprocessor units that facilitate, for instance, the coherent reshaping of photons in time and frequency must be available. Note that these do not necessarily have to fulfill the more stringent demands of fault-tolerant quantum computation to be practical and, therefore, as we show, can be realized with current technology.

In this paper, we introduce a practical framework for photonic quantum information science. Our framework exploits temporal modes (TMs) of single photon statesfield-orthogonal broadband wave-packet states-that to date have not been demonstrated to enable a viable basis for quantum information encoding. In particular, we complement existing knowledge with all missing building blocks, which are needed to demonstrate that TMs satisfy the three major requirements for the implementation of the photonic subsystems of large-scale quantum networks: firstly, for the preparation of good signal carriers, appropriate resource states have to be generated and completely characterized with high reliability and flexibility; secondly, the subsequent processing of quantum information in coprocessor units requires that controlled operations can be implemented; finally, efficient detection schemes, which enable faithful information read-out, must be available.

We expect that the TM framework for photonic quantum information will open avenues towards the realization of practical QIS applications. One such application is the boson sampler [2-6], which, though not on par with the requirements for fault-tolerant quantum computation, may soon show performance beyond the capabilities of stateof-the-art classical computers, which are pushed to their limits by linear optical networks with about 100 modes, of which only 30 are occupied. Our new TM paradigm may offer improved methods to construct large networks with reduced switching losses, which are currently thought to be 
the main limiting factor when considering the scalability of photonic quantum processing [7].

In the following, we first introduce the basic concepts of our framework by formally defining TMs and their use as an information-encoding basis. Then, we briefly review the current state of the art of generating TMs with ultrafast parametric down-conversion (PDC), where we outline why existing sources do not yet fulfill the requirements for QIS with TMs. After this, we highlight recent developments in TM manipulation, which serve as the starting point for the definition of the complete TM framework. The key enabling findings for this are our recent results, which introduce means for sorting TMs with high efficiency and selectivity in excess of $99.5 \%$. This high efficiency of the "quantum pulse gate" (QPG) operation can be achieved by dispersion-engineered, multistage frequency conversion driven by spectro-temporal shaped laser control pulses. We then present new concepts and components, which enable the establishment of the complete TM framework. In particular, we design the flexible generation of entangled resource states of arbitrary, user-defined dimension, we introduce TM quantum-state tomography of single-photon as well as photon-pair states to verify the successful state generation, and we establish concrete applications for QIS. We show that all operations necessary to implement photonic coprocessors and quantum communication applications can be implemented with TMs. We conclude the paper with a discussion of the experimental challenges and limitations of our framework.

\section{FUNDAMENTAL CONCEPTS}

Starting from a very general point of view, we note that light has four degrees of freedom (DOF), any of which could be used to encode quantum information: these are the helicity and the three components of the momentum vector. In a beamlike geometry these may be stated as polarization, transverse mode profile (encompassing two DOFs), and energy (that is, frequency). From these DOFs, polarization is most widely applied in quantum information processing. The generation of polarization-entangled Bell states [8] as resource states is nowadays an established experimental method. Two orthogonal polarization modes can easily be separated by means of using polarizing beam splitters, and proper gate operations are readily implemented with linear optical elements such as wave plates, (polarizing) beam splitters, and detectors. However, polarization intrinsically spans a mere two-dimensional Hilbert space, and thus cannot exploit the true potential of QIS, which, in certain cases such as quantum key distribution (QKD), benefits from higher-dimensional Hilbert spaces $[9,10]$.

The second DOF, transverse mode profile, has received considerable attention recently, as it has become apparent that the orbital-angular-momentum (OAM) states of light are a useful basis for encoding information [11-13] and can be efficiently sorted with time-stationary linear optical elements [14]. They have been used recently to demonstrate, for instance, enhanced security and bitrate in quantum communication [15-17]. Still, the OAM basis has three drawbacks limiting its current value for some QIS applications: first, it is inherently incompatible with existing single-mode fiber networks because information is encoded onto different spatial field distributions; second, it is susceptible to medium perturbations such as turbulence, which affects free-space links; and third, the generation of OAM states with a tailored structure, for instance, a welldefined number of modes, is as of yet an unsolved problem.

Only recently has the final DOF of light—energy, that is frequency-been recognized as an underutilized resource for QIS. Because frequency and time are conjugate variables, we call a set of overlapping but orthogonal broadband wave-packet modes by the name "temporal modes." In a coherent-beam-like or single-transversemode guided wave geometry, TMs form a complete basis for representing an arbitrary state in the energy degree of freedom [18]. TMs overlap in time and frequency, yet are field orthogonal. In this respect, they are analogous to transverse spatial modes, yet they possess distinct advantages. Since all TMs "live" inside the same spatial field distribution, they are naturally suited for use with highly efficient and experimentally robust waveguide devices and existing single-mode fiber networks. In addition, they are insensitive to stationary or slowly varying medium perturbations such as linear dispersion, due to their overlapping spectra, making them suitable for real-world applications.

While the TM concept applies to any states of light (e.g., squeezed quadrature states $[19,20])$, we restrict ourselves to single-photon states to keep this paper concise and readable. In this context, TMs are a complete mode set for expanding the electromagnetic field and, in addition, can be regarded as a complete set of quantum states for single photons.

\section{A. Temporal modes for single-photon states}

For a fixed polarization and transverse field distribution (e.g., in a beamlike geometry), a single-photon quantum state in a specific TM can be expressed as a coherent superposition of a continuum of single-photon states in different monochromatic modes:

$$
\left|A_{j}\right\rangle=\int \frac{d \omega}{2 \pi} f_{j}(\omega) \hat{a}^{\dagger}(\omega)|0\rangle .
$$

Here, $\hat{a}^{\dagger}(\omega)$ is the standard monochromatic creation operator and $f_{j}(\omega)$ is the complex spectral amplitude of the wave packet. By Fourier transform, this same state can be expressed as a coherent superposition over many possible "creation times," and then reads 


$$
\left|A_{j}\right\rangle=\int d t \tilde{f}_{j}(t) \hat{A}^{\dagger}(t)|0\rangle \equiv \hat{A}_{j}^{\dagger}|0\rangle,
$$

where we use the definition

$\hat{a}^{\dagger}(\omega)=\int d t e^{i \omega t} \hat{A}^{\dagger}(t), \quad \hat{A}^{\dagger}(t)=\int \frac{d \omega}{2 \pi} e^{-l \omega t} \hat{a}^{\dagger}(\omega)$.

In Eq. (2), $\tilde{f}_{j}(t)$ is the temporal shape of the wave packet [defined as the Fourier transform of $\left.f_{j}(\omega)\right]$ and $\hat{A}^{\dagger}(t)$ creates a photon at time $t$. We also define a so-called broadband-mode operator

$$
\hat{A}_{j}^{\dagger}=\int d t \tilde{f}_{j}(t) \hat{A}^{\dagger}(t)=\frac{1}{2 \pi} \int d \omega f_{j}(\omega) \hat{a}^{\dagger}(\omega),
$$

which creates the wave-packet state $\left|A_{j}\right\rangle$. In Fig. 1, we exemplarily plot the first three members of a TM basis, chosen for illustration to be a family of Hermite-Gaussian functions of frequency. With this, it is possible to express every single-photon temporal wave-packet quantum state $|\Psi\rangle$ in a basis of TMs as a superposition of wave-packet states,

$$
|\Psi\rangle=\sum_{j=0}^{\infty} c_{j} \hat{A}_{j}^{\dagger}|0\rangle,
$$

with complex-valued expansion coefficients $c_{j}$.

We want to highlight that, although they fully overlap in polarization, space, frequency, and time, TMs are orthogonal with respect to a frequency (time) integral,

$$
\begin{aligned}
& \frac{1}{2 \pi} \int d \omega f_{j}^{*}(\omega) f_{k}(\omega)=\int d t \tilde{f}_{j}^{*}(t) \tilde{f}_{k}(t)=\delta_{j k} . \\
& \left|A_{j}\right\rangle=\frac{1}{2 \pi} \int d \omega f_{j}(\omega) \hat{a}^{\dagger}(\omega)|0\rangle \quad\left|A_{j}\right\rangle=\int d t \tilde{f}_{j}(t) \hat{A}^{\dagger}(t)|0\rangle \\
& f_{j}(\omega) \bigcap_{\omega} j=0 \\
& f_{j}(\omega) \downarrow \bigcap_{\omega} j=1 \\
& f_{j}(\omega) \bigcap \bigcup_{\omega} j=2
\end{aligned}
$$
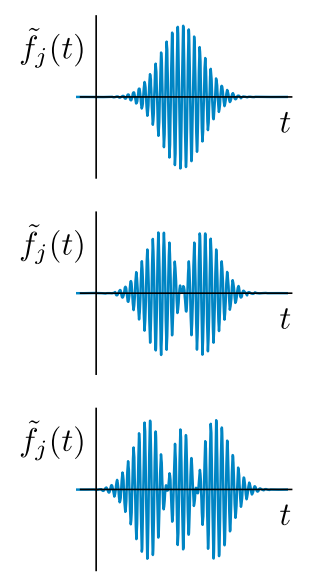

FIG. 1. First three members of a TM basis in the frequency domain (left) and the time domain (right).
They also obey bosonic commutation relations [18,21],

$$
\left[\hat{A}_{i}, \hat{A}_{j}^{\dagger}\right]=\delta_{i j}
$$

just as do the well-known monochromatic creation operators.

\section{B. Quantum information encoding with TMs}

Deploying TMs for quantum information encoding is an appealing prospect, because TMs span an infinitedimensional Hilbert space. This has been shown to facilitate increased information capacity per photon and increased security in quantum communication [15-17] when compared to two-dimensional encoding. The carriers of information in a $d$-dimensional Hilbert space are typically called "qudits."

We define a TM qudit as a coherent superposition of $d$ TM states:

$$
|\psi\rangle_{\mathrm{TM}}^{d}=\sum_{j=0}^{d-1} \alpha_{j}\left|A_{j}\right\rangle .
$$

To highlight the formal similarity of TMs with other encoding bases, we start by discussing TM qubits. The most common implementation of a photonic qubit is the polarization qubit, which can be written as $|\psi\rangle=$ $\alpha|H\rangle+\beta|V\rangle$. Here, $|H\rangle$ and $|V\rangle$ denote horizontal and vertical polarization, respectively, and $|\alpha|^{2}+|\beta|^{2}=1$. Commonly, a polarization qubit is represented as a point on the surface of a Poincaré sphere, as sketched in Fig. 2(a).

In analogy to this, the definition of a TM qubit requires two orthogonal states with which we associate the logical "0" and " 1. " Without loss of generality, we can consider zeroth-order and first-order Hermite-Gaussian functions of frequency to define the TMs, labeled $\curvearrowright$ and $\sim$, and consequently write
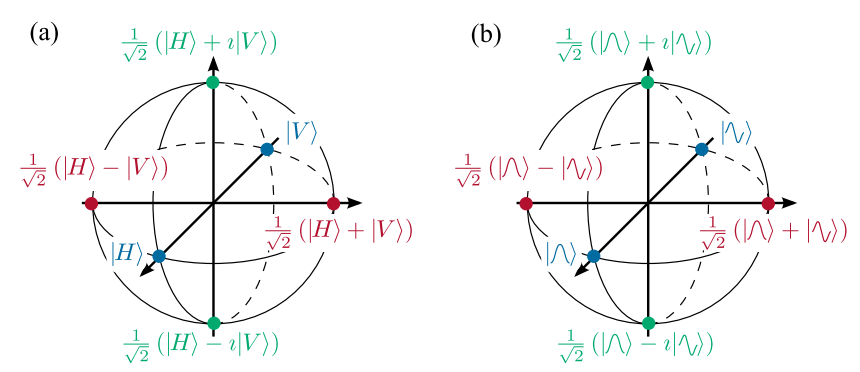

FIG. 2. (a) Poincaré sphere. The logical "0" and " 1 " of a polarization qubit can be encoded in any two diametrically opposite points on the sphere. Typically, horizontal and vertical polarization are deployed. (b) Bloch sphere for TM qubits. Any two orthogonal TMs and their coherent superpositions may be used to encode TM qubits. In this example, the TMs are zerothand first-order Hermite-Gaussian pulses. 


$$
|0\rangle \equiv|\frown\rangle, \quad|1\rangle \equiv|\sim\rangle
$$

Then, a TM qubit is given by

$$
|\psi\rangle_{\mathrm{TM}} \equiv \alpha|\curvearrowright\rangle+\beta|\sim\rangle,
$$

where again $|\alpha|^{2}+|\beta|^{2}=1$. Similar to polarization qubits, the TM qubit is best visualized as a point on the surface of a Bloch sphere, as shown in Fig. 2(b).

\section{Mutually unbiased bases}

Sets of bases, for which the overlap between a basis vector of one basis with any basis vector from any of the other bases has the same absolute value, are called mutually unbiased bases (MUBs) [22]. They lie at the heart of QIS applications such as quantum key distribution [23] or quantum state tomography [24]. The physical meaning of MUBs is the following: if a certain quantum state is an eigenstate of one basis, then a measurement in any other MUB yields a uniformly random result yielding no information. Using polarization states, the three sets of Stokes vectors denoting horizontal and vertical, diagonal and antidiagonal, as well as left- and right-circular light form the typically used MUBs.

For the case of the aforementioned TM qubit from Fig. 2(b), the basis modes of the three possible MUBs are indicated by the different colors and we explicitly plot them in Fig. 3. The color coding corresponds to Fig. 2(b). If the qubit was given by $\curvearrowright$, measuring in either the "red" or "green" basis results in "0" (upper row) or " 1 " (lower row) with a probability of $50 \%$.

The challenge for TMs is the implementation of a device that facilitates a mode-selective measurement, where the phase coherence plays a particularly important role. For a polarization qubit, an appropriate combination of wave
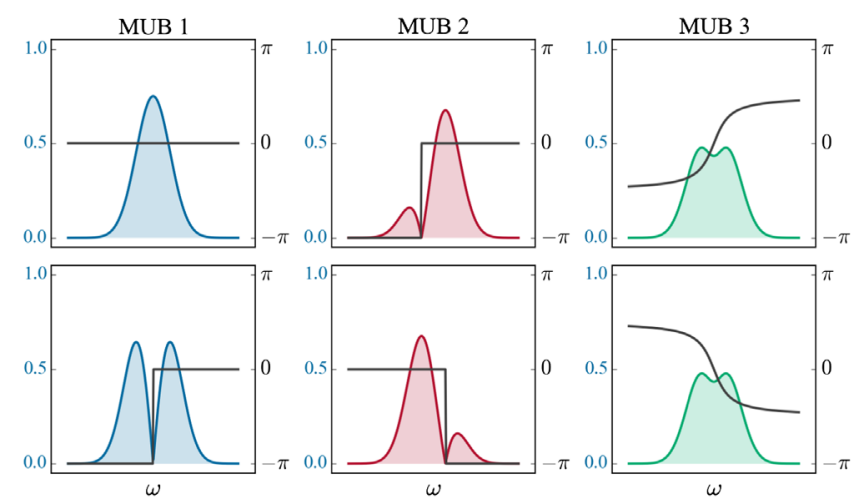

FIG. 3. The columns show the three MUBs for a TM qubit, with the fundamental TM shapes being a zeroth- and first-order Hermite-Gaussian pulse, respectively. The colored areas are the spectral amplitude, whereas the dark lines are the spectral phases of the TMs; the color coding corresponds to Fig. 2(b). Note that in this case, the qubit is encoded in the leftmost basis. plates and polarizing beam splitters readily accomplishes the projection onto the respective basis sets. For TMs, the situation is more complicated, since time-stationary operations are not sufficient for mode selectivity and so-called quantum pulse gates have to be employed [25-28]. We return to this point below, where we briefly review the solution to the mode-sorting problem.

\section{STATE OF THE ART}

In this section, we briefly summarize the current state of the art in generating and manipulating TM states. Typically, the former is realized with parametric down-conversion, whereas the latter can be achieved by deploying TMselective quantum pulse gates.

\section{A. TM structure of photon pair states}

Today, parametric down-conversion in optical waveguides is the workhorse for the generation of photon-pair and heralded single-photon states. Notably, PDC generates quantum states with a rich intrinsic TM structure, when ultrafast pulses are deployed as pump [29]. This structure is decoupled from the transverse spatial mode, which is solely determined by the waveguide geometry. It is encoded in the so-called joint spectral amplitude (JSA) of the PDC $f\left(\omega_{s}, \omega_{i}\right)$, which can be written as $[30,31]$

$$
f\left(\omega_{s}, \omega_{i}\right)=\alpha\left(\omega_{s}, \omega_{i}\right) \cdot \phi\left(\omega_{s}, \omega_{i}\right)
$$

Here, $\alpha\left(\omega_{s}, \omega_{i}\right)$ is the pump-envelope function, which encompasses energy conservation and the spectrum of the pump pulses, and $\phi\left(\omega_{s}, \omega_{i}\right)$ is the phase-matching function, which describes momentum conservation and depends on the medium dispersion.

With that, we denote the photon-pair component of the generated state,

$$
|\psi\rangle_{\mathrm{PDC}}=\int d \omega_{s} d \omega_{i} f\left(\omega_{s}, \omega_{i}\right) \hat{a}^{\dagger}\left(\omega_{s}\right) \hat{b}^{\dagger}\left(\omega_{i}\right)|0,0\rangle,
$$

where $\hat{a}^{\dagger}\left(\omega_{s}\right)$ and $\hat{b}^{\dagger}\left(\omega_{i}\right)$ are standard monochromatic creation operators for signal and idler photons.

A decomposition of the JSA into two sets of uniquely defined TM basis functions $\left\{f^{(s)}\left(\omega_{s}\right)\right\}$ and $\left\{f^{(i)}\left(\omega_{i}\right)\right\}$, which exhibit pairwise correlations such that

$$
f\left(\omega_{s}, \omega_{i}\right)=\sum_{k=0}^{\infty} \sqrt{\lambda_{k}} f_{k}^{(s)}\left(\omega_{s}\right) f_{k}^{(i)}\left(\omega_{i}\right)
$$

reveals the underlying TM structure of the PDC state [29]. Here, the expansion coefficients are normalized according to $\sum_{k} \lambda_{k}=1$. We graphically show this expansion for a typical, nonengineered PDC in Fig. 4(a). 

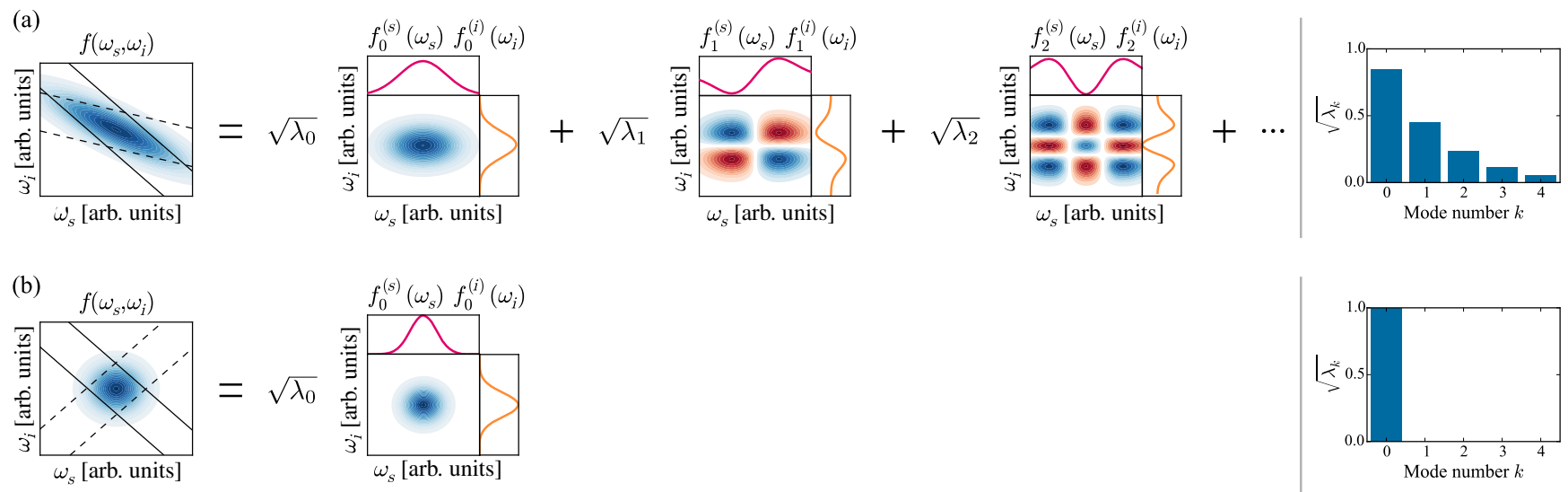

FIG. 4. (a) Representation of a general PDC process. The leftmost panel shows the JSA $f\left(\omega_{s}, \omega_{i}\right)$, which is the product of pump envelope function (black solid lines) and the phase-matching function (black dashed lines). This function is decomposed into two sets of TMs $\left\{f^{(s)}\left(\omega_{s}\right)\right\}$ and $\left\{f^{(i)}\left(\omega_{i}\right)\right\}$ with weighting coefficients $\sqrt{\lambda_{k}}$. In the central part, we plot the first three TM pairs. The rightmost panel shows the distribution of expansion coefficients $\sqrt{\lambda_{k}}$. (b) A dispersion-engineered PDC process excites only one pair of TMs. The JSA does not exhibit any correlations between signal and idler photons. The distribution of weighting coefficients $\sqrt{\lambda_{k}}$ consequently exhibits only a single entry greater than zero.

From Eqs. (12) and (13), we obtain

$$
|\psi\rangle_{\mathrm{PDC}}=\sum_{k=0}^{\infty} \sqrt{\lambda_{k}}\left|A_{k}, B_{k}\right\rangle
$$

where we again use the broadband mode operators from Eq. (4). This expression shows that the PDC excites pairs of TM states $\left|A_{k}\right\rangle$ and $\left|B_{k}\right\rangle$ with a relative weight of $\sqrt{\lambda_{k}}$.

For the special case of a dispersion-engineered PDC that excites only a single pair of TMs (see, for instance, Refs. [32-37]), the state from Eq. (14) reduces to $|\psi\rangle_{\mathrm{PDC}}=\left|A_{0}, B_{0}\right\rangle$. This situation is shown in Fig. 4(b). In this case, by detecting the photon created in one channel, one heralds the single-photon state in the other channel in a known, pure TM. We note, however, that this is not sufficient for generating resource states for QIS applications. On the one hand, the general PDC state has an inadequate structure, because the number of TMs in the state cannot be precisely controlled. On the other hand, the single-TM state does not constitute an entangled resource state, which is a necessary requirement for different QIS applications.

\section{B. Coherent manipulation of the TM structure of single-photon states}

A major requirement for realizing QIS with TMs is the coherent manipulation of a state in the TM basis. This can be achieved by deploying so-called quantum pulse gates $[25,27,28,38]$. Note that although we restrict our discussions to three-wave mixing implementations of QPGs here, all results can be generalized to four-wave mixing. The underlying physical process of a QPG based on three-wave mixing is dispersion-engineered sum-frequency generation inside a nonlinear optical waveguide, where one photon from an ultrafast pump pulse and a "red" quantum signal fuse into a "green" converted output photon. Here, red and green describe two well-separated frequency bands, for instance, $1535 \mathrm{~nm}$ (red) and $557 \mathrm{~nm}$ (green), respectively [26]. An adaption of this approach for use with continuousvariable quantum states has been proposed in Ref. [20]. In four-wave mixing implementations, two nondegenerate pump pulses are used, which facilitate smaller frequency shifts of single photons as compared to using three-wave mixing [39-41].

An ideal QPG that is mode matched to the TMs of the source as defined above acts on an arbitrary single-photon input state $|\psi\rangle_{\text {in }}$ of the form Eq. (8) according to

$$
|\psi\rangle_{\text {out }}=\hat{Q}_{i}^{(\eta)}|\psi\rangle_{\text {in }}
$$

with

$$
\begin{aligned}
\hat{Q}_{i}^{(\eta)}= & \mathbb{1}-\left|A_{i}\right\rangle\left\langle A_{i}|-| C\right\rangle\langle C| \\
& +\cos \theta_{i}\left(\left|A_{i}\right\rangle\left\langle A_{i}|+| C\right\rangle\langle C|\right) \\
& +\sin \theta_{i}\left(|C\rangle\left\langle A_{i}|-| A_{i}\right\rangle\langle C|\right) .
\end{aligned}
$$

The cosine term preserves either of the two states of interest, while the sine term "swaps" them with efficiency $\sin ^{2}\left(\theta_{i}\right)$. The first three terms enforce unitarity. This expression is a family of unitary transformations on the single-photon state space composed of two nonoverlapping subspaces (here, frequency bands): one spanned by the TM states $\left|A_{j}\right\rangle$, and a single TM state $|C\rangle$ occupying the other. It has an elegant interpretation: the QPG acts as a quantum mechanical beam splitter, which operates on TMs instead of polarization or spatial modes. As detailed in Refs. [38,42], the blue pump pulse spectrum $\alpha(\omega)$ defines 
(a)

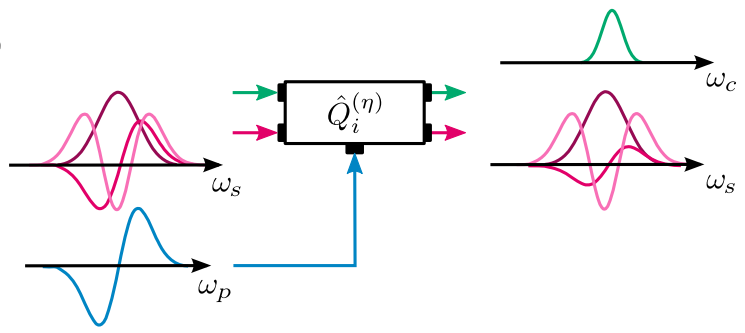

(b)

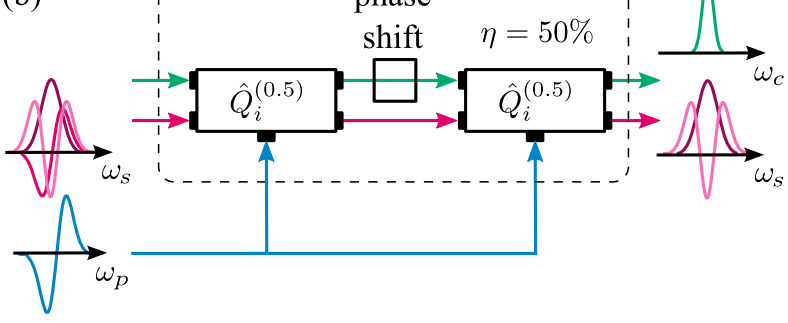

FIG. 5. (a) Schematic of the QPG operation. The shape of the blue pump pulse selects one TM from the "red" input signal and converts it to the "green" output with an efficiency of $\eta$. All other signal TMs are completely transmitted. The index $i$ labels the addressed input TM. (b) A Mach-Zehnder- or Ramsey-like configuration of two successive QPGs with an efficiency of $50 \%$ each overcomes the time-ordering limitations of a single QPG and facilitates the selection and conversion of a single TM with an efficiency of $100 \%$.

the targeted "red" input TM state $\left|A_{i}\right\rangle$ that is selected and converted to the "green" output state $|C\rangle$ with an efficiency given by $\eta=\sin ^{2}\left(\theta_{i}\right)$. Note that the QPG can also select superpositions of TM states, when the pump pulses are shaped accordingly. The parameter $\theta_{i}$ describes the strength of the QPG operation and can be tuned with the pump pulse energy, although the shapes of the "red" and "green" modes will change slightly for different values of $\theta_{i}$, due to timeordering corrections [43-45] (i.e., the input and output TMs are not identical). For genuine QPG operation, $\theta_{j}=0$ for $j \neq i$; that is, all TMs that are not addressed are completely transmitted. This situation is sketched in Fig. 5(a).

From Eq. (16) we see two things. First, the QPG converts any targeted input state $\left|A_{i}\right\rangle$ into the same output state $|C\rangle$. This is important in light of large network architectures, because it facilitates interference between formerly orthogonal TM states after the QPG operation. Second, the QPG can also be operated "backwards." In this case, it accepts one single input state $|C\rangle$, which is coherently reshaped to an arbitrary output TM state $\left|A_{i}\right\rangle$. This allows the treatment of the $|C\rangle$ frequency band as a buffer, or "processing" state space, and allows one to perform arbitrary linear operations on TM qudits that reside in the $\left\{\left|A_{j}\right\rangle\right\}$ space using combinations of QPGs, as we show below.

A measure to quantify the operation fidelity of a QPG is the so-called temporal mode selectivity [27],

$$
S=\frac{\sin ^{4}\left(\theta_{i}\right)}{\sum_{j=0}^{\infty} \sin ^{2}\left(\theta_{j}\right)} \leq 1,
$$

which measures the ratio between the squared conversion efficiency of the selected mode and the conversion efficiencies of all modes. A mode selectivity of 1 characterizes perfect single-TM operation, whereas a mode selectivity of 0 signifies a total absence of modal selectivity.

It has been shown that the single-stage QPG realization from Fig. 5(a) cannot exceed a mode selectivity of $S=0.85$ due to the effects of time ordering, which lead to a temporal multimode behavior at conversion efficiencies exceeding $90 \%[43,44]$. This limitation can be overcome by utilizing a two-stage Mach-Zehnder- or Ramsey-like setup of two successive QPGs with an efficiency of 50\% each, which are driven by the same pump pulse shape [27,28]. We sketch this in Fig. 5(b).

In the two-stage QPG a single photon in the target TM will be converted into an equal superposition of a "green" and a "red" mode by the first stage, and will then be coherently fully frequency shifted or backconverted in the second stage depending on an externally applied phase shift to the device. The nontarget TM components of the photon will not participate in the interferometric conversion process due to their vanishingly small per-stage conversion efficiencies, and will effectively transparently pass through the device. The need for phase coherence across the two stages can be met by deriving the two pump pulses from the same master pulse. In a specific configuration [27,28], this method also eliminates the temporal distortion in the shapes of the "red" and "green" modes due to time-ordering effects, which enables the cascading of QPGs without the need for inter-QPG compensatory TM reshaping. Note that the overall operation of the two QPGs is again collectively described by Eq. (16) and that we use the simplified sketch from Fig. 5(a) for reasons of convenience from here on. Various overall efficiency values can now be achieved by tuning the interferometric phase shift in between the two stages [Fig. 5(b)] instead of changing the pump power.

In a recent experiment, the implementation of a singlestage QPG with a TM selectivity of $80 \%$ at a conversion efficiency of $\eta=87 \%$ when operated at the single-photon level has been demonstrated [26].

Note that alternative approaches to TM-selective sumfrequency generation are studied in Refs. [46-48], which forego group-velocity matching. Although potentially simpler from an experimental point of view, these approaches cannot generally reach high selectivities as defined above [43].

\section{COMPLETING THE TOOL KIT FOR A TM QIS FRAMEWORK}

In this section, we introduce the missing components, which enable our TM framework. In particular, these are 
the generation of TM states with an arbitrary, user-defined dimension and their verification using single-photon and photon-pair TM tomography. Thereafter, we show that ideal QPGs can be used to implement linear-optics singleand photon-pair quantum operations.

\section{A. TM engineering and TM Bell states}

Typical QIS applications require at least the faithful generation of Bell states. In the following, we demonstrate how this can be accomplished for TMs by combining in a very natural way a dispersion-engineered PDC with pulseshaping techniques, which are well established in the fields of ultrafast optics and coherent control (for a good review, see Ref. [49]).

To this end, we consider shaped pump pulses with Hermite-Gaussian spectra given by

$$
\alpha\left(\omega_{s}, \omega_{i}\right)=\frac{1}{\sqrt{n ! \sqrt{\pi} 2^{n} \sigma}} H_{n}\left(\frac{\Delta \omega}{\sigma}\right) \exp \left[-\frac{(\Delta \omega)^{2}}{2 \sigma^{2}}\right] .
$$

Here, $\Delta \omega=\omega_{p}-\omega_{s}-\omega_{i}$ is the frequency mismatch between the pump, signal, and idler fields, $H_{n}(x)$ is a Hermite polynomial of order $n$, and $\sigma$ is the spectral $1 / e$ width of the pump spectral intensity.

Figure 6(a) shows an engineered PDC that is driven by a first-order Hermite-Gaussian pump pulse. The JSA decomposes into

$$
f\left(\omega_{s}, \omega_{i}\right)=\frac{1}{\sqrt{2}}\left[f_{0}^{(s)}\left(\omega_{s}\right) f_{0}^{(i)}\left(\omega_{i}\right)+f_{1}^{(s)}\left(\omega_{s}\right) f_{1}^{(i)}\left(\omega_{i}\right)\right] .
$$

This result can be interpreted such that the PDC comprises exactly two pairs of TMs with equal excitation probability. Consequently, we write the generated photonpair state as

$$
\begin{aligned}
|\psi\rangle_{\mathrm{PDC}} & \approx \frac{1}{\sqrt{2}}\left(\left|A_{0}, B_{0}\right\rangle+\left|A_{1}, B_{1}\right\rangle\right) \\
& =\frac{1}{\sqrt{2}}\left(\left|\frown_{\mathrm{s}}, \sim_{\mathrm{i}}\right\rangle+\left|\sim_{\mathrm{s}}, \frown_{\mathrm{i}}\right\rangle\right)
\end{aligned}
$$

where the graphical representation in the second line highlights the shapes of the individual signal and idler TMs. This state is a TM $\left|\psi^{+}\right\rangle$Bell state, which is a fundamental resource for QIS applications.

In Fig. 6(b), we consider a second-order HermiteGaussian pump pulse. The decomposition of the resulting JSA shows that the generated state comprises exactly three TM pairs. Although the relative weights are not evenly distributed anymore, the dimensionality of the state is well defined. Further increasing the order of the pump HermiteGaussian pulse successively adds additional TM pairs to the structure of the generated state.

In this way it is possible to generate high-dimensional photonic states with an unprecedented degree of control. We emphasize again that all TMs "live" inside the same transverse spatial waveguide mode, which makes our approach exceptionally robust and guarantees experimental simplicity.

\section{B. Photon TM-state tomography}

With the ability to generate TM states with arbitrary dimension, the missing element to render a QIS framework based on TMs feasible is the verification of the state generation. To this end, we require TM-state tomography, where the challenge is to retrieve the (complex-valued) entries of a quantum state's density matrix in a basis of
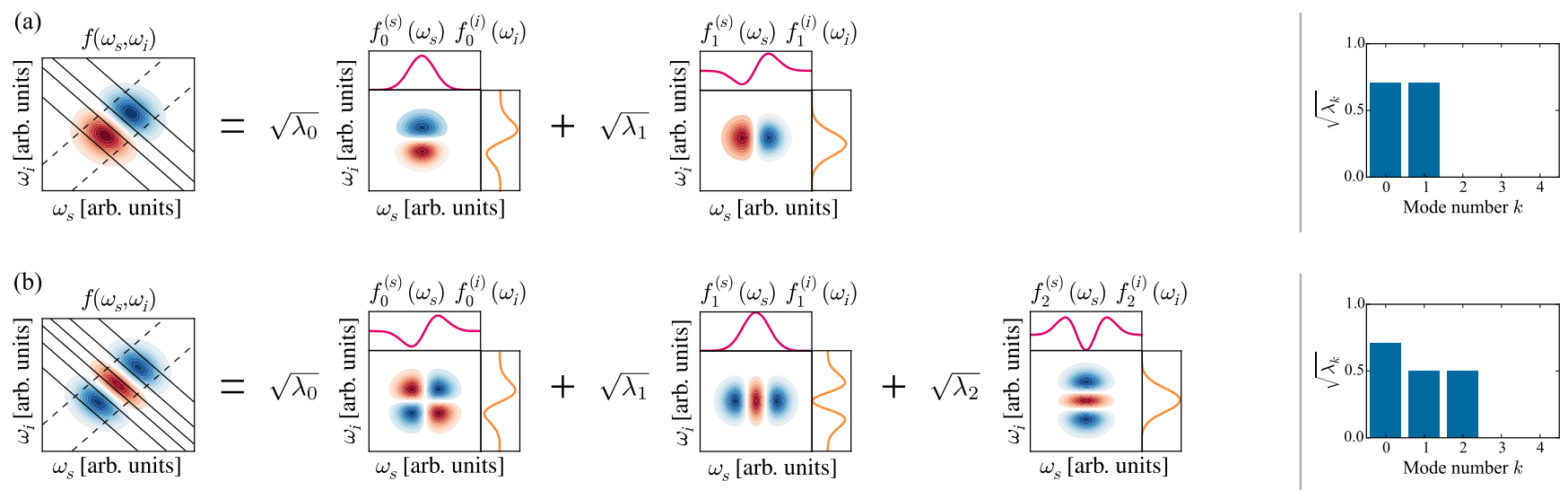

FIG. 6. (a) When pumping a dispersion-engineered PDC with a first-order Hermite-Gaussian pulse, the resulting JSA (left) has a negative part signified by the red color. Note that the pump envelope function is again denoted by solid black lines, whereas the phasematching function is shown as dashed black lines. A decomposition of this JSA yields exactly two pairs of TMs (center) with similar expansion coefficients (right). Hence, the generated state is a TM Bell state. (b) By further increasing the order of the Hermite-Gaussian pump, it is possible to successively add TM pairs to the generated state. This state features an extremely well-defined dimensionality, although the relative weights of the modes become unbalanced. 
(a)

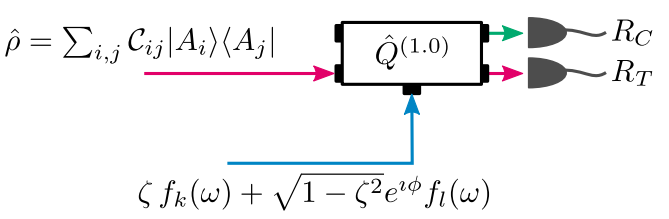

(b)

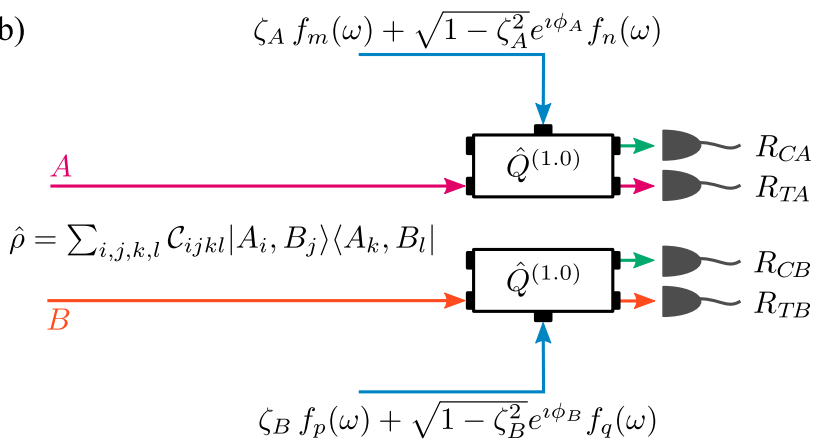

FIG. 7. (a) TM state tomography of a single-photon state with density matrix $\hat{\rho}$. Both transmitted and converted output of the QPG are detected with single-photon detectors. (b) Generalized scheme for the TM tomography of a biphoton state. Photons " 1 " and " 2 " are sent to two different QPGs, and the transmitted and converted outputs are detected with single-photon detectors.

TMs. This differs from polarization-state tomography because of the higher dimensionality of the TM-state space. For an arbitrary single-photon state, the density matrix is given by

$$
\hat{\rho}=\sum_{i, j} \mathcal{C}_{i j}\left|A_{i}\right\rangle\left\langle A_{j}\right|
$$

with associated TMs $\left\{f_{i}(\omega)\right\}$. This state can be analyzed with a QPG, which selects a coherent superposition of TMs given by $\zeta f_{k}(\omega)+\sqrt{1-\zeta^{2}} e^{l \phi} f_{l}(\omega)$, where $\zeta \in[0,1]$, as shown in Fig. 7(a). This function is defined by the shape of the pump pulse the QPG is "programmed" with. Detecting both the converted output and the transmitted light with single-photon detectors, we measure the average converted count rates $R_{C}$ and $R_{T}$, respectively, which are related to elements of the input density matrix by

$$
\frac{R_{C}}{R_{C}+R_{T}}=\zeta^{2} \mathcal{C}_{k k}+\left(1-\zeta^{2}\right) \mathcal{C}_{l l}+2 \operatorname{Re}\left[\zeta \sqrt{1-\zeta^{2}} e^{\imath \phi} \mathcal{C}_{l k}\right]
$$

From this expression, we see that for $\zeta=0$ and $\zeta=1$, we directly obtain $\mathcal{C}_{k k}$ and $\mathcal{C}_{l l}$, respectively. To retrieve the complex coefficient $\mathcal{C}_{l k}$, we set $\zeta=(1 / \sqrt{2})$ and evaluate the counts for $\phi=0$ and $\phi=(\pi / 2)$. By extension, we also obtain $\mathcal{C}_{k l}$ and thus a complete subset of matrix coefficients of the density matrix $\hat{\rho}$. In this way, the complete density matrix or an experimentally feasible subset thereof can be sampled. It is important to note that any chosen portion of the density matrix can be "directly" measured in this way without reconstructing the entire state. This is true only for a QPG that can achieve unit selectivity, although without high selectivity, the elements can still be found up to an unknown normalization constant. This would necessitate measuring the entire matrix (or making small-magnitude assumptions about the unmeasured coefficients).

This procedure can be generalized to certain biphoton states as sketched in Fig. 7(b). A general two-photon state in two different spatial modes (with photon labels $A$ and $B$ ) may be expressed in two sets of TM bases as

$$
\hat{\rho}=\sum_{i, j, k, l} \mathcal{C}_{i j k l}\left|A_{i}, B_{j}\right\rangle\left\langle A_{k}, B_{l}\right| .
$$

The two photons are analyzed with two separate QPGs, which select TMs given by $\zeta_{A} f_{m}(\omega)+\sqrt{1-\zeta_{A}^{2}} e^{\imath \phi_{A}} f_{n}(\omega)$ and $\zeta_{B} f_{p}(\omega)+\sqrt{1-\zeta_{B}^{2}} e^{l \phi_{B}} f_{q}(\omega)$, respectively. Then we employ four single-photon detectors labeled $C A, T A, C B$, and $T B$, as shown in Fig. 7(b). We can then measure coincidence rates between pairs of detectors (say between $C A$ and $C B$, denoted by $R_{C A, C B}$, and so on). The following expression of such coincidence rates,

$$
\frac{R_{C A, C B}}{R_{C A, C B}+R_{C A, T B}+R_{T A, C B}+R_{T A, T B}},
$$

can be expressed in terms of the biphoton density matrix elements as

$$
\begin{aligned}
& \zeta_{A}^{2} \zeta_{B}^{2} \mathcal{C}_{m p p m}+\left(1-\zeta_{A}^{2}\right)\left(1-\zeta_{B}^{2}\right) \mathcal{C}_{n q q n}+\zeta_{A}^{2}\left(1-\zeta_{B}^{2}\right) \mathcal{C}_{m q q m}+\left(1-\zeta_{A}^{2}\right) \zeta_{B}^{2} \mathcal{C}_{n p p n} \\
& 2 \operatorname{Re}\left\{e^{\imath \phi_{A}} \zeta_{A} \sqrt{1-\zeta_{A}}\left[\zeta_{B}^{2} \mathcal{C}_{m p p n}+\left(1-\zeta_{B}^{2}\right) \mathcal{C}_{m q q n}\right]+e^{\imath \phi_{B}} \zeta_{B} \sqrt{1-\zeta_{B}}\left[\zeta_{A}^{2} \mathcal{C}_{m p q m}+\left(1-\zeta_{A}^{2}\right) \mathcal{C}_{n q q n}\right]\right. \\
& \left.+\zeta_{A} \zeta_{B} \sqrt{1-\zeta_{A}} \sqrt{1-\zeta_{B}}\left(e^{l\left(\phi_{A}+\phi_{B}\right)} \mathcal{C}_{m p q n}+e^{l\left(\phi_{A}-\phi_{B}\right)} \mathcal{C}_{m q p n}\right)\right\}
\end{aligned}
$$

Cycling through the parameter space $\left(\zeta_{1,2}, \phi_{1,2}\right) \in$ $\{(1,-),(0,-),(1 / \sqrt{2}, 0),(1 / \sqrt{2}, \pi / 2)\} \quad$ as well as varying the indices $(m, n, p, q)$ will reveal any desired set of coefficients from the two-photon density matrix.

\section{QIS APPLICATIONS}

In this section, we combine the different building blocks to detail several QIS applications, which can be realized in the TM framework and highlight its versatility. We first 
consider photon TM purification and TM reshaping, then move on to quantum communication scenarios and conclude with considerations on single-qubit gate operations and cluster-state generation. Note that we discuss the technical challenges that have to be faced when implementing these applications in detail in the following section.

\section{A. Photon TM "purification"}

Let us consider an application, which requires either a photon-pair at very specific wavelengths or a choice of nonlinear material, such that it is not possible to directly implement a dispersion-engineered PDC source that generates only a single pair of TMs, but instead a general PDC state as sketched in Fig. 4(a). In this case, people typically resort to spectrally narrow intensity filtering to facilitate the heralding of approximately pure single photons, thus discarding the greater portion of the generated photon pairs [50,51]. Our TM tool kit provides a more efficient and elegant solution to this problem, which additionally facilitates the heralding of genuinely pure broadband single photons from a correlated source, such as shown in Fig. 4(a).

We assume the general PDC state from Eq. (14) and detect one of the photons, say, photon $A$, with an unfiltered single-photon detector as sketched in Fig. 8(a). This heralds photon $B$ with a reduced density matrix that is given by

$$
\hat{\rho}_{B}=\sum_{k=0}^{\infty} \lambda_{k}\left|B_{k}\right\rangle\left\langle B_{k}\right|,
$$

which is generally a mixed state with purity $\mathcal{P}=\sum_{k} \lambda_{k}^{2}$.

On the other hand, we can send photon $A$ to a QPG, which acts as a complex spectral-amplitude shape "filter" that selects a single TM $f_{i}^{(s)}\left(\omega_{s}\right)$ with efficiency $\eta$, and detect only the converted output. In this case, a successful detection heralds photon $B$, which is in a pure state with corresponding density matrix,

$$
\hat{\rho}_{B}=\left|B_{i}\right\rangle\left\langle B_{i}\right|,
$$

as sketched in Fig. 8(b) [25]. Note that this "purification" comes at the cost of a lower heralding rate, which is

(a)

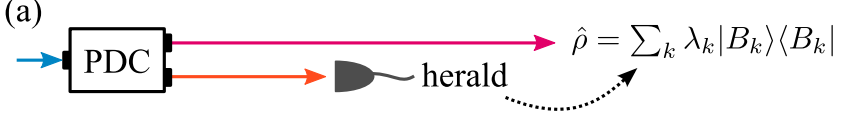

(b)

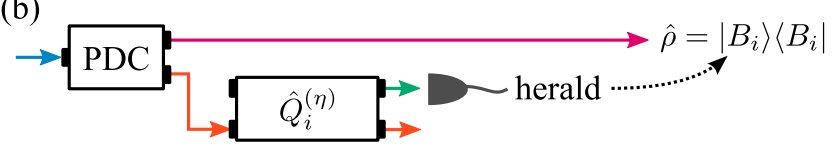

FIG. 8. (a) Non-mode-selective detection of one PDC photon generally projects its sibling into a mixed state. (b) Deploying a QPG to herald a single TM yields a pure heralded broadband photon at the cost of a lowered heralding rate. reduced by the factor $\lambda_{i}$. Still, the advantage is that a photon in a desired TM can be created, rather than simply a spectrally filtered photon.

As a side remark, although we restrict our analysis to photon-pair states, the TM framework can be directly applied to continuous variable states. In this context, a particularly important non-Gaussian operation is TMselective photon subtraction from a multimode state, which is required for entanglement distillation [52]. It is based on the same operation as the photon TM purification, but uses a QPG that is intentionally operated at very low conversion efficiency [20].

\section{B. Single-photon TM reshaping}

Large-scale networks require an efficient interfacing between distinct nodes. For different photon sources, this means that the photons have to be made indistinguishable. For coupling photons to solid-state systems, this means that the TM of the photons has to match the acceptance TM of the system. In both cases, a coherent TM reshaping of the photons is preferable to other filtering operations, since the latter introduce prohibitive losses. In Fig. 9, we sketch a TM reshaper: A first QPG converts the "red" input photon-which we implicitly assume to be pure and thus TM single mode - to the "green" channel; a second QPG is then used to backconvert the photon to the "red" channel. However, here we match the shape of the bright pump pulse to the required TM and by this reshape the photon. Note that the reshaped mode does not have to be a mode from the original photon TM basis, which is indicated by the label $A$ (as opposed to a numeric label) of the QPG operation in the figure. The complete reshaping operation can then be written as

$$
|\psi\rangle_{A}=\hat{Q}_{A}^{(1.0)} \hat{Q}_{0}^{(1.0)}\left|A_{0}\right\rangle=\hat{Q}_{A}^{(1.0)}|C\rangle=-\left|A_{A}\right\rangle,
$$

where we assume the original photon to be in the TM state $\left|A_{0}\right\rangle$ and the overall phase of the output state can be neglected. The operators $\hat{Q}_{i}^{(1.0)}$ are the QPG operators from Eq. (16). In principle, arbitrary reshaping is possible in this way. Note that a reshaping of the green TM can be realized by tailoring the phase-matching function of the QPG [53-55]. In this way, an adapted interface between photons at telecommunication wavelengths and specific quantum memories can be realized with a single QPG.

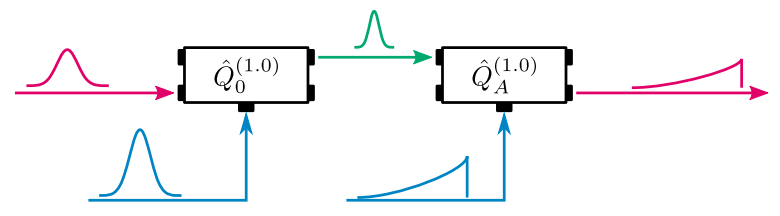

FIG. 9. TM reshaping of a single photon. A QPG first converts the red single photon to the green channel. A second QPG then reshapes the photon during backconversion. 


\section{Quantum communication}

Another important aspect of QIS is quantum communication, where quantum information is transmitted between distant parties, by convention called Alice and Bob. To this end, information has to be encoded at Alice's location and decoded and read out at Bob's location. Deploying the aforementioned devices and methods, a QC system based on TMs can be readily set up.

Here, we discuss two approaches to realizing this. The first approach utilizes different TMs as different communication channels and thus relies on TM multiplexing. Note that in this approach, information is not encoded in the TMs but in another degree of freedom, for instance, the polarization. The second approach directly encodes the information in arbitrary superpositions of single-photon TMs, and thereby can implement genuine high-dimensional QC.

The use of TMs for channel multiplexing would be distinguished from conventional time- or frequency-based optical multiplexing, which use either separated short pulses or narrow spectral windows to define different information channels. Such schemes have recently been proposed in the general context of QIS as well $[56,57]$. However, they are not based on genuinely field-orthogonal modes, which translates to a lower "packing density" of signal channels in time-frequency space to ensure approximate orthogonality. A fundamental advantage of our TM approach is that it is intrinsically based on genuinely fieldorthogonal wave-packet modes, which provide in-principle zero cross talk between mode channels, while densely packing these modes in time-frequency phase space.

In QC, for a TM multiplexing a scheme to work, adddrop functionality is essential. Using the QPG, both

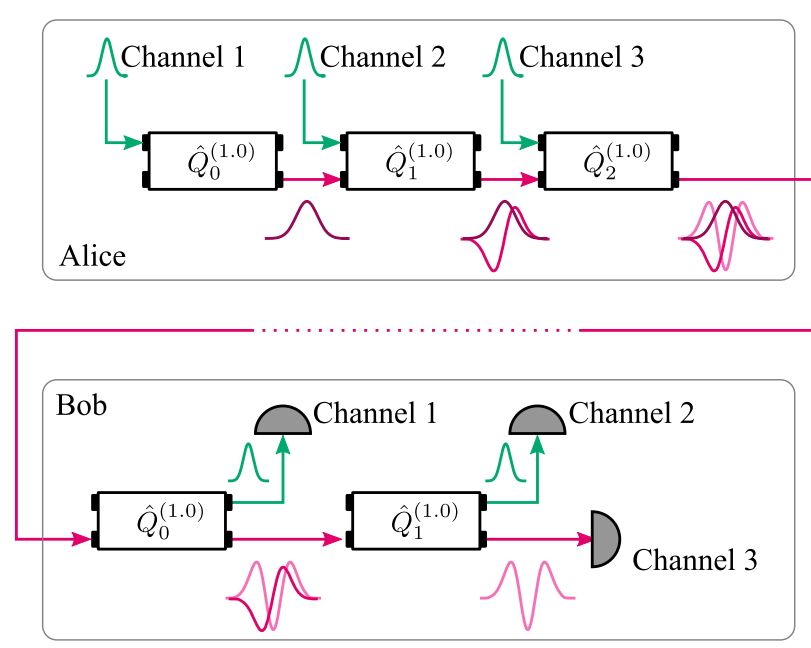

FIG. 10. In a TM multiplexing scenario, Alice uses orthogonal TMs as independent channels, which are sent to Bob in one single physical fiber. He demultiplexes the channels with QPGs and reads out the information. The QPGs are being employed as TM multiplexers (Alice) and demultiplexers (Bob) on a single-mode optical channel. operations can be implemented as sketched in Fig. 10(a). On Alice's side, a succession of QPGs adds different channels to the communication line. This is possible due to the TM-selective operation of the QPG, which reshapes the "green" input $f^{(c)}(\omega)$ into the desired "red" TM $f_{i}^{(s)}(\omega)$. At the same time, the existing "red" TMs $f_{j}^{(s)}(\omega)$ with $j \neq i$ are not affected. Note that this operation mode of the QPG has been referred to as quantum pulse shaper earlier [38]. After transmission, Bob deploys a cascade of QPGs to demultiplex the different channels into separate ports, from which the information is read out [46].

The second approach, high-dimensional QC, is appealing in light of quantum key distribution applications, where the goal is to establish a secure encryption key between Alice and Bob. Deploying TMs, the implementation of a generalized BB84 protocol [23] becomes possible. To clarify this procedure, we first sketch the realization of the original BB84 protocol using two TM MUBs instead of polarization in Fig. 11(a). Alice randomly prepares one of the four possible basis states with a QPG and sends it to Bob. Bob in turn randomly chooses the measurement basis of his QPG and directly detects both output ports, which then correspond to 0 and 1. Thereafter, Alice and Bob publicly announce their preparation and measurement bases and keep only those events when both coincide. Sacrificing a part of the so-retained key, Alice and Bob can uncover an eavesdropper by the $25 \%$ error he or she inevitably introduces.

This scheme is readily extended to $d$ dimensions. We illustrate this for the case of $d=4$, which is depicted in Fig. 11(b). In this case, five MUBs and thus a total of 20 possible basis states exist, from which Alice randomly chooses one. The four basis states of each MUB now encode logical 0 to 3 . In the figure, we use the first four Hermite-Gaussian pulses as the "mother" basis from which "daughter" MUBs are created. Again, Alice transmits the chosen state to Bob who performs the read-out in a randomly chosen basis. Note, however, that Bob now requires three QPGs to completely separate the four basis states of the MUBs. More generally, Bob requires $d-1$ QPGs for a basis of size $d$. There are two major advantages to high-dimensional encoding schemes in QC. On the one hand, high-dimensional encoding facilitates a higher information capacity per photon, and thus leads to a reduction in the overall number of required photons. On the other hand, it has been shown that high-dimensional encoding can increase the security of quantum key distribution, due to a larger error that is introduced by a potential eavesdropper when intercepting the transmission $[9,10]$.

\section{Quantum computation}

In this section, we discuss two routes towards quantum computation enabled by the completion of the TM tool kit. First, we consider linear optical quantum computation (LOQC), where TM qubits propagate through 
(a)

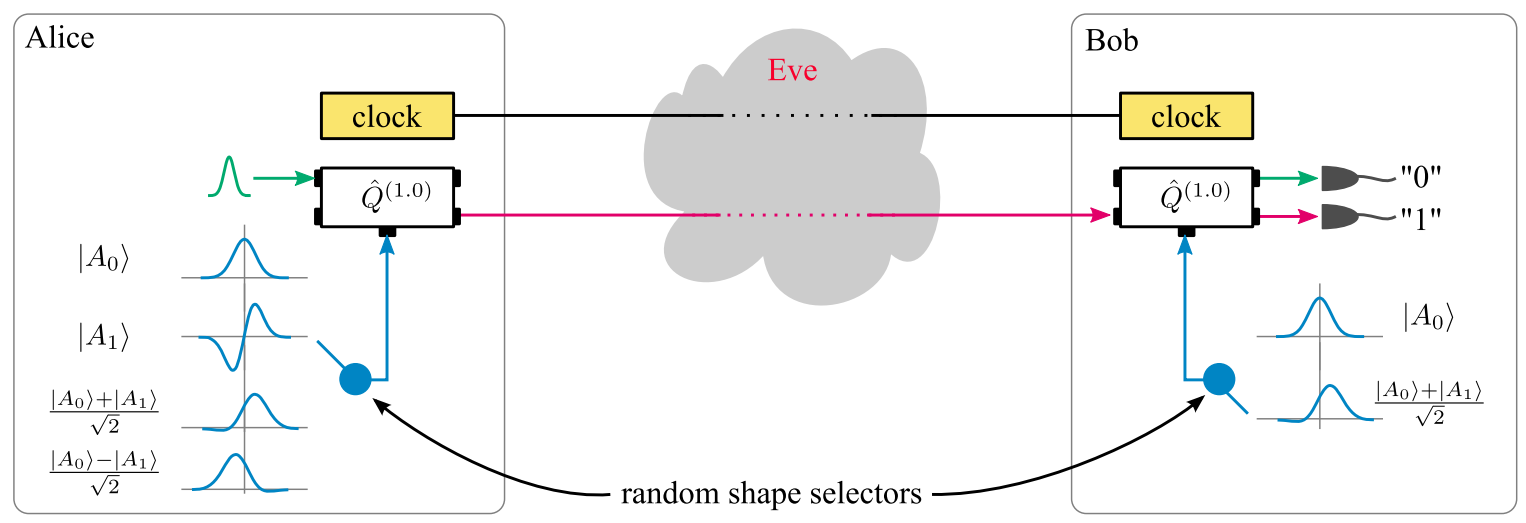

(b)

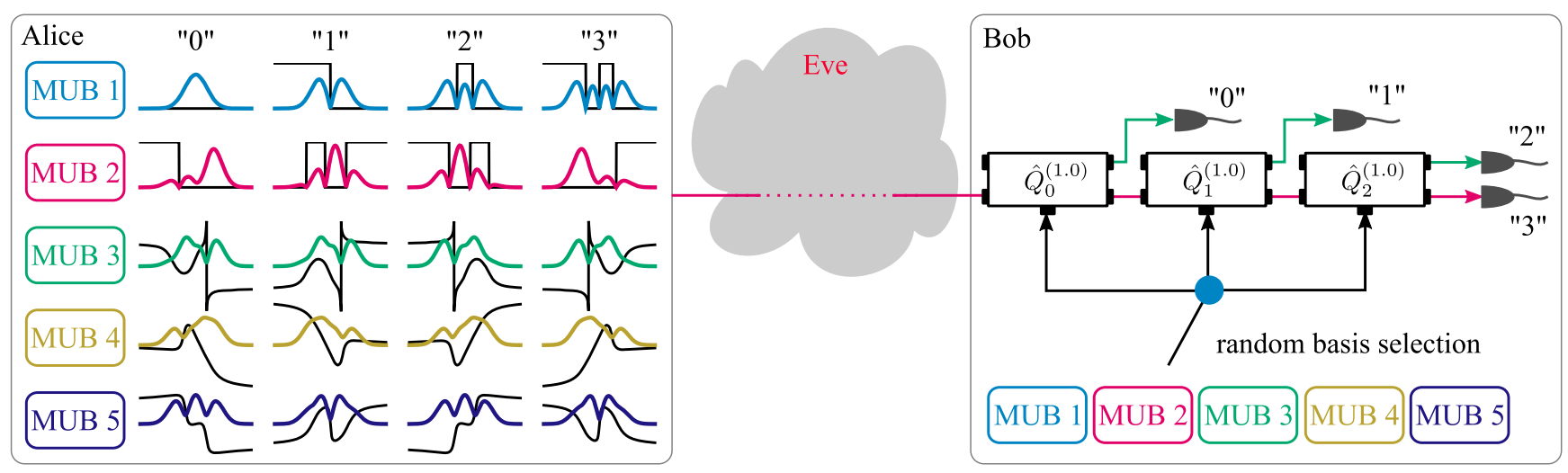

FIG. 11. (a) Implementation of the BB84 QKD protocol with TMs. Alice randomly prepares one of four possible basis states and sends it to Bob, who randomly measures in one of two MUBs. The two outputs of Bob's QPG correspond to 0 and 1 . (b) Generalized BB84 in a four-dimensional encoding scheme. Alice randomly prepares one of the 20 possible basis states. Bob chooses randomly one of the five MUBs to measure. Note that in this case he requires three QPGs to fully resolve the four possible basis states of each MUB.

a linear-optical network and are subject to single- and twoqubit operations, which define the computation algorithm. Then, we investigate cluster-state quantum computation, where multiple TM qubits are fused in a specific way to create a graph state with a tailored entanglement structure. Then, measurements of the nodes (photons) of the cluster state implement the computation algorithm, the result of which can be read out from the remaining nodes. Although universal photonic quantum computation is beyond today's technological capabilities [7], the required operational building blocks can be realized with TMs.

Since in this paper we focus on three-wave mixing implementations of QPGs, we are effectively restricted to one single "green" output TM state $|C\rangle$, though we allow for a complete set of "red" input TM states $\left|A_{i}\right\rangle$. Consequently, the input states are treated as the primary qudit information "register" space, and the output channel will play the role of a "processing" space. Note that this behavior gives rise to the question of whether QPGs are sufficient to realize all of the necessary operations for quantum computation. We show in the following that they are.

\section{1. $L O Q C$}

In LOQC, deterministic two-qubit operations are provably impossible. However, arbitrary single-qubit operations can be implemented with a combination of QPGs. For this, we require two special cases of the QPG operation from Eq. (16). First, a QPG with a conversion efficiency of $100 \%$, and second, a QPG with a conversion efficiency of $50 \%$. They are represented by operators

$$
\hat{Q}_{i}^{(1.0)}=\mathbb{1}-\left|A_{i}\right\rangle\left\langle A_{i}|-| C\right\rangle\langle C|+| C\rangle\left\langle A_{i}|-| A_{i}\right\rangle\langle C|
$$

and

$$
\begin{aligned}
\hat{Q}_{i}^{(0.5)}= & \mathbb{1}-\left|A_{i}\right\rangle\left\langle A_{i}|-| C\right\rangle\langle C| \\
& +\frac{1}{\sqrt{2}}\left(\left|A_{i}\right\rangle\left\langle A_{i}|+| C\right\rangle\langle C|\right) \\
& +\frac{1}{\sqrt{2}}\left(|C\rangle\left\langle A_{i}|-| A_{i}\right\rangle\langle C|\right) .
\end{aligned}
$$


In Fig. 12, we show how these operations driven by the proper pump shapes can be sequentially combined with channel-dependent phase shifts, which shift the phase only in the "green" processing space and are shown as "green" boxes, to implement the following single-qubit operations (up to an overall phase) on the $\left\{\left|A_{0}\right\rangle,\left|A_{1}\right\rangle\right\}$ space:

(a) Hadamard gate,

$$
\hat{H}=\frac{\left|A_{0}\right\rangle+\left|A_{1}\right\rangle}{\sqrt{2}}\left\langle A_{0}\right|+\frac{\left|A_{0}\right\rangle-\left|A_{1}\right\rangle}{\sqrt{2}}\left\langle A_{1}\right| ;
$$

(b) Pauli- $X$ gate (type I, II),

$$
\hat{X}=\left|A_{1}\right\rangle\left\langle A_{0}|+| A_{0}\right\rangle\left\langle A_{1}\right| ;
$$

(a)

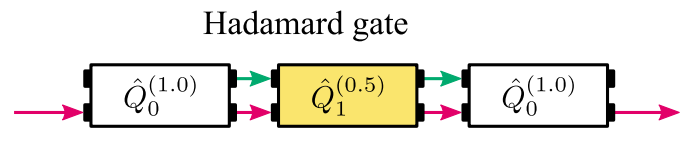

(b)

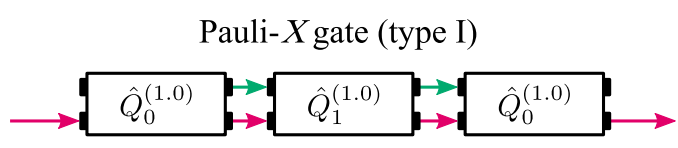

Pauli- $X$ gate (type II)

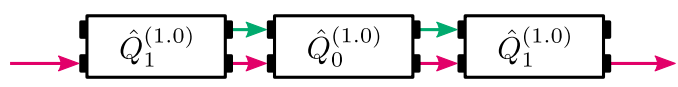

(c)

Pauli- $Y$ gate (type I)

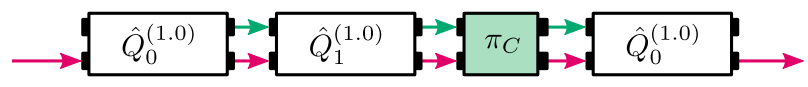

Pauli- $Y$ gate (type II)

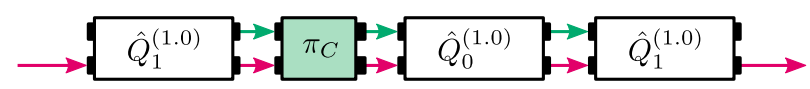

(d)

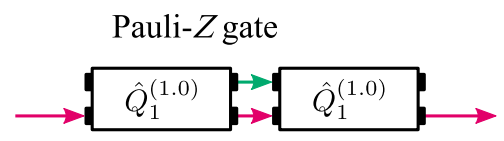

(e)

Phase-shift gate

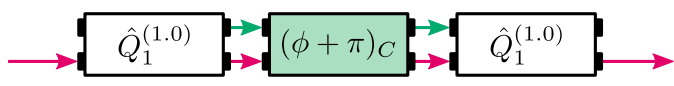

FIG. 12. Implementation of single-qubit gates for LOQC using QPGs with 100\% conversion efficiency (white boxes), QPGs with $50 \%$ conversion efficiency (yellow boxes), and phase shifts of the green $|C\rangle$ TM (green boxes). Note that both the Pauli- $X$ gate and the Pauli- $Y$ gate have two possible experimental implementations, which differ in the order in which the red TMs $f_{0}^{(s)}(\omega)$ and $f_{1}^{(s)}(\omega)$ are addressed. (c) Pauli- $Y$ gate (type I, II),

$$
\hat{Y}=-\imath\left|A_{1}\right\rangle\left\langle A_{0}|+\imath| A_{0}\right\rangle\left\langle A_{1}\right| ;
$$

(d) Pauli- $Z$ gate,

$$
\hat{Z}=\left|A_{0}\right\rangle\left\langle A_{0}|-| A_{1}\right\rangle\left\langle A_{1}\right|
$$

(e) phase-shift gate,

$$
\hat{\phi}=\left|A_{0}\right\rangle\left\langle A_{0}\left|+e^{\imath \phi}\right| A_{1}\right\rangle\left\langle A_{1}\right| .
$$

These realizations rely on only two different pump shapes, corresponding to the "red" TMs $f_{0}^{(s)}(\omega)$ and $f_{1}^{(s)}(\omega)$, which encode the logical 0 and 1 . The phase-shift gate can be simplified, if the phase $(\phi+\pi)$ is imprinted onto one of the two pump pulses. Then, the channeldependent phase shift can be omitted.

Note that the "green" channel is used only internally, whereas the input and output channels are the red TMs. This greatly reduces the challenge of maintaining phase relations between different frequency bands. It also eliminates the phase-coherence requirement for pump pulses across different red-channel-to-red-channel single-qubit gates, only requiring it for pump pulses internal to any given single-qubit gate. Additionally, the sequential steps can, in principle, be fabricated in monolithic devices, which promises a compact and robust implementation with building blocks that are well suited to be used in integrated networks.

We also emphasize that, in a manner similar to Ref. [58], any single qudit operation can be realized with a concatenation of the single-qubit operations outlined in this section. Then, the pump shapes have to be chosen such that the single-qubit gates operate on every twodimensional subspace of the qudit space successively.

\section{Cluster-state quantum computation}

Finally, we consider the generation of discrete variable cluster states based on TMs. To efficiently grow cluster states from a supply of resource Bell pairs, we require several operations. Assuming that we already have a stock of linear cluster states that we want to merge into twodimensional cluster states, we need local Hadamard transformations and projective measurements [59]. We have already shown how these can be implemented with TMs. More important is the ability to generate linear cluster states from Bell pairs. In order to do so, we have to rely on qubit fusion. A general method that facilitates this for polarization qubits has been introduced by Browne and Rudolph [60], where it was referred to as Type-I fusion. Here, we adapt this scheme to operate on TM qubits as defined in Eq. (10). 


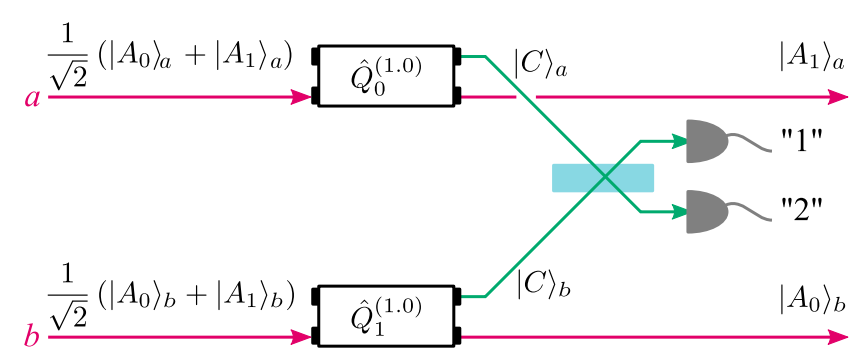

FIG. 13. Two TM qubits in spatial beams $a$ and $b$ can be fused with two QPGs, which select different "red" TM components from the qubits and selectively frequency convert them. Then, the "green" outputs of the QPGs are interfered at a 50/50 beam splitter (blue rectangle) and detected with detectors 1 and 2. For more information, see the text.

Two qubits in spatial beams $a$ and $b$ are sent to two QPGs, as sketched in Fig. 13. The QPGs implement the operation $\hat{Q}_{0}^{(1.0)}$ on qubit $a$ and $\hat{Q}_{1}^{(1.0)}$ on qubit $b$, respectively. This means that the "red" TMs $f_{0, a}^{(s)}(\omega)$ and $f_{1, b}^{(s)}(\omega)$ are converted to the "green" TMs $f^{(c)}(\omega)_{a, b}$. The two green channels are interfered on a balanced beam splitter behind the QPGs to erase any distinguishing information and the beam splitter output ports are detected by detectors 1 and 2 . The successful detection of a single "green" photon heralds the successful qubit fusion operation, which can be written in terms of Kraus operators,

$$
\hat{O}_{1,2}=\frac{1}{\sqrt{2}}\left(| A _ { 0 } \rangle _ { b } \left\langleA _ { 0 } | _ { a } \langle A _ { 0 } | _ { b } \mp | A _ { 1 } \rangle _ { b } \left\langle\left.A_{1}\right|_{a}\left\langle\left. A_{1}\right|_{b}\right),\right.\right.\right.
$$

where the sign depends on whether detector 1 or 2 fires. The state after a successful fusion is given by

$$
|\psi\rangle_{\text {fused }}=\frac{1}{\sqrt{2}}\left(\left|A_{0}\right\rangle_{b} \mp\left|A_{1}\right\rangle_{a}\right),
$$

which, as expected, again denotes a qubit state. Note that the two parts of the fused qubit can be deterministically combined into a single spatial mode with the add-drop functionality of the QPG discussed in the context of quantum communication.

\section{CHALLENGES}

In this section, we detail the challenges one faces when implementing QIS applications based on TMs. While photonic quantum information systems are ideal for serving as intermediary between memory, interaction, and detection resources, they come with known challenges. Most notably, the absence of any direct photon-photon interaction limits all-optical quantum information processing to nondeterministic logic gates [61] or cluster-state measurement schemes [62]. When compared with opticalpolarization or beam-path encoding of quantum information, the proposed TM encoding brings additional challenges, which need to be overcome in order to take advantage of the large in-principle benefits of using TMs for QIS: their relative immunity from channel dispersion and their compatibility with quantum memories in hybrid QIS systems, where efficient coupling into and out of disparate devices is highly dependent on temporal mode matching which can, in principle, be achieved with TM reshaping.

For this, the limiting factor is the bandwidth $\Delta \nu_{\mathrm{PM}}$ of the phase-matching function of the QPG, which determines the minimal bandwidth of the reshaped TM. For the QPG presented in Ref. [26], the spectrum of the "green" TM had a FWHM of $\Delta \lambda_{g}=0.14 \mathrm{~nm}$, corresponding to a bandwidth of $\Delta \nu_{g} \approx 135 \mathrm{GHz}$, which equals $\Delta \nu_{\mathrm{PM}}$ [38]. We can calculate the narrowest possible phase-matching bandwidth of a QPG based on a lithium niobate waveguide with uniform periodic poling. The maximum waveguide length is limited by the size of commercial lithium niobate crystals to around $L_{\max } \approx 90 \mathrm{~mm}$. Using this number, the resulting phase-matching bandwidth is calculated to be $\Delta \nu_{\mathrm{PM}} \approx 9.7 \mathrm{GHz}$, which is close to the maximum bandwidth of state-of-the-art quantum memories based on Raman interaction in warm Cs vapor of $9.2 \mathrm{GHz}$ [63]. In addition, recent results on manipulating the phase-matching function by manipulating the periodic poling pattern of waveguides [53-55] hold the promise for a future decrease of the effective phase-matching bandwidth. Thus, deploying QPGs as interfaces between flying and stationary qubits is a realistic vision.

An additional complication when interfacing flying and stationary qudits is the required multimode capability of the quantum memory. The Raman memory mentioned above, for instance, can store only a single TM [64]. However, it has recently been shown that a concatenation of several Raman-type memories can overcome this limitation and store high-dimensional TM states [65]. This result is a promising step towards the realization of high-dimensional hybrid quantum networks and facilitates the seamless integration of quantum memories into the TM framework.

A further challenge is the achievable loss budget for a QIS application based on TMs. In this context, we highlight again that all TMs live inside the same spatial mode and thus feature low-loss transmission through standard singlemode fibers. In addition, waveguide to fiber coupling with efficiencies exceeding $92 \%$ has been demonstrated [66]. Finally, waveguide propagation losses as low as $0.016 \mathrm{~dB} / \mathrm{cm}$ in titanium-indiffused lithium niobate waveguides can be realized with state-of-the-art technology [67]. In total, we find an insertion loss of roughly $1.0 \mathrm{~dB}$ for coupling from a fiber to a 90-mm-long QPG and back to a fiber. In this case, the main losses arise from the fiber couplings. We note that this challenge is not singular to the TM framework, but rather a challenge that the whole field of integrated quantum photonics has to face. Although current loss numbers are still prohibitively high, a 
significant increase in waveguide to fiber coupling efficiency can be expected in the coming years, which will alleviate this situation.

Let us now focus on the realistically achievable number of TMs and thus the dimensionality of the accessible Hilbert space. With increasing mode order, the complexity as well as the spectral extent of TMs increases. Hence, the number of modes will be bounded, on the one hand, by the resolution of the deployed pulse shapers for pump pulses and, on the other hand, by the maximum spectral bandwidth of single-TM operation of the QPGs. For the demonstrator from Ref. [26], the maximum spectral bandwidth can be calculated to be around $25 \mathrm{~nm}$ for an input signal centered around $1550 \mathrm{~nm}$. For larger bandwidths, the group-velocity dispersion inside the waveguide becomes non-negligible and the process is not TM single-mode anymore. Let us then assume that the TM states are generated with a PDC in a potassium titanyl phosphate waveguide as presented in Refs. [36,37]. Then, the FWHM of the fundamental TM is around $5.0 \mathrm{~nm}$. In this case, 10 TMs can be addressed with a selectivity in excess of $95 \%$. A simple optimization of the PDC bandwidth and the length of the QPG waveguide increases this number to 20 TMs. Note that this is the limit of only the particular realization of a QPG based on lithium niobate waveguides. Investigating other materials with a more favorable group-velocity dispersion behavior can yield an even higher mode number.

Concerning the resolution of state-of-the-art pulse shapers, we note that spatial light modulators with up to 4096 pixels are commercially available. Paired with proper imaging optics, these devices are capable of shaping TMs of order 100 with a fidelity of more than $99.9 \%$. With respect to spatial light modulators, we also note their current limited switching speeds, which are typically in the order of few tens of $\mathrm{kHz}$. These impose an upper limit on the switching speed of QIS applications. Again, this challenge does not only affect the TM framework, but also QIS based on transverse spatial modes, which also relies on spatial light modulators as key elements.

Now, we consider the fidelity of the LOQG gate operations. In Ref. [28], Reddy et al. investigate the mode selectivity of two-stage and multistage approaches to realizing QPGs. They found that in a two-stage QPG, the maximum selectivity is $S \approx 98.46 \%$, which translates to a maximum gate fidelity of around $95.4 \%$, since every LOQC gate consists of three QPGs. This value cannot compete with requirements on fault-tolerant LOQC, but may facilitate small coprocessing operations with only few gates. In addition, by increasing the number of stages in the QPG, the selectivity asymptotically approaches one. Thus, there is a trade-off between the TM selectivity and the total internal losses of a gate operation, which has to be evaluated in light of specific applications' requirements.

Finally, let us turn our attention to the synchronization of the time-dependent, active components driven by shaped laser pulses in a TM framework. The timing requirements may be more severe when using TMs instead of other encoding bases, because the TM scheme relies essentially on temporal orthogonality, which is degraded under time jitter. To overcome this timing challenge over long-distance transmissions, we envision the use of weak coherent "pilot" pulses, which when amplified at the receiver can serve as a timing reference, a pump pulse, and a transmissionmedium-induced linear-dispersion compensator, all in one.

In general, we find that, as with all burgeoning frameworks for optical QIS, the use of TMs will require significant investments in integrated device fabrication technology and timing electronics. TMs also share with other frameworks the need for efficient single-photon detection and lossless programmable optical routing. Ultimately, TM-based schemes might have to rely on performance gains from single-mode networkability and higher-dimensionality, supplemented by their accommodation of broadband quantum memories, to outperform other optical QIS frameworks.

\section{CONCLUSION}

We show that TMs of single-photon states form an appealing framework for QIS. Formally, they are comparable with transverse spatial field modes, but have distinct advantages over spatial modes: they are naturally compatible with waveguide technology, making them ideal candidates for integration into existing communication networks, and they are not affected by typical medium distortions such as linear dispersion, which renders them robust basis states for real-world applications. Still, TMs are as yet an underused resource for QIS.

In this paper, we demonstrate that QIS based on TMs is feasible with current technology. We introduce a novel method for the generation of photon pair states comprising a user-defined number of TMs, which facilitates, in particular, the generation of TM Bell states. This method relies on the combination of dispersion-engineered PDC with classical pulse shaping for the pump pulses of the process. We then propose TM tomography of single-photon and photon-pair states as building blocks for a QIS framework based on TMs.

Having established the necessary basis, we move on to the implementation of QIS applications. With small photonic coprocessing units embedded into large-scale hybrid quantum networks in mind, we first focus on TM "purification" and TM reshaping. Thereafter, we discuss quantum communication based on TMs, where we present two approaches: a TM multiplexing approach, where different TMs represent independent channels, and a highdimensional TM QKD scenario, where the information is encoded into the order of the TMs. Finally, we demonstrate that any single qudit operation can be implemented with a succession of properly adjusted QPGs. We conclude the applications section with a scheme for TM cluster state 
generation which highlights the versatility of the TM framework.

Finally, we discuss in detail technical challenges that must be faced when implementing QIS based on TMs. We expect that the introduction of this new framework will open novel research avenues in both fundamental and applied QIS.

\section{ACKNOWLEDGMENTS}

D. V. R. and M. G. R. were supported by the National Science Foundation through ENG-EPMD and PHYSQIS. B. B. and C.S. acknowledge financial support by the Deutsche Forschungsgemeinschaft (DFG) via Sonderforschungsbereich TRR 142.

[1] H. J. Kimble, The Quantum Internet, Nature (London) 453, 1023 (2008).

[2] S. Aaronson and A. Arkhipov, The computation complexity of linear optics, in Proceedings of the 43rd Annual ACM Symposium on the Theory of Computing (STOC'11), San Jose, CA, 2011 (ACM, New York, 2011), pp. 333-342.

[3] J. B. Spring, B. J. Metcalf, P. C. Humphreys, W. S. Kolthammer, X.-M. Jin, M. Barbieri, A. Datta, N. Thomas-Peter, N. K. Langford, D. Kundys, J. C. Gates, B. J. Smith, P. G. R. Smith, and I. A. Walmsley, Boson Sampling on a Photonic Chip, Science 339, 798 (2013).

[4] M. A. Broome, A. Fedrizzi, S. Rahimi-Keshari, J. Dove, S. Aaronson, T. C. Ralph, and A. G. White, Photonic Boson Sampling in a Tunable Circuit, Science 339, 794 (2013).

[5] A. Crespi, R. Osellame, R. Ramponi, D. J. Brod, E. F. Galvão, N. Spagnolo, C. Vitelli, E. Maiorino, P. Mataloni, and F. Sciarrino, Integrated Multimode Interferometers with Arbitrary Designs for Photonic Boson Sampling, Nat. Photonics 7, 545 (2013).

[6] M. Tillmann, B. Dakić, R. Heilmann, S. Nolte, A. Szameit, and P. Walther, Experimental Boson Sampling, Nat. Photonics 7, 540 (2013).

[7] Y. Li, P. C. Humphreys, G. J. Mendoza, and S. C. Benjamin, Resource Costs for Fault-Tolerant Linear Optical Quantum Computing, arXiv:1504.02457 [Phys. Rev. X (to be published)].

[8] P. G. Kwiat, K. Mattle, H. Weinfurter, A. Zeilinger, A. V. Sergienko, and Y. Shih, New High-Intensity Source of Polarization-Entangled Photon Pairs, Phys. Rev. Lett. 75, 4337 (1995).

[9] H. Bechmann-Pasquinucci and W. Tittel, Quantum Cryptography Using Larger Alphabets, Phys. Rev. A 61, 062308 (2000).

[10] N. J. Cerf, M. Bourennane, A. Karlsson, and N. Gisin, Security of Quantum Key Distribution Using d-Level Systems, Phys. Rev. Lett. 88, 127902 (2002).

[11] A. Mair, A. Vaziri, G. Weihs, and A. Zeilinger, Entanglement of the Orbital Angular Momentum States of Photons, Nature (London) 412, 313 (2001).

[12] J. Leach, B. Jack, J. Romero, A. K. Jha, A. M. Yao, S. Franke-Arnold, D. G. Ireland, R. W. Boyd, S. M. Barnett, and M.J. Padgett, Quantum Correlations in Optical
Angle-Orbital Angular Momentum Variables, Science 329, 662 (2010).

[13] A. C. Dada, J. Leach, G. S. Buller, M. J. Padgett, and E. Andersson, Experimental High-Dimensional Two-Photon Entanglement and Violations of Generalized Bell Inequalities, Nat. Phys. 7, 677 (2011).

[14] G. C. G. Berkhout, M. P. J. Lavery, J. Courtial, M. W. Beijersbergen, and M. J. Padgett, Efficient Sorting of Orbital Angular Momentum States of Light, Phys. Rev. Lett. 105, 153601 (2010).

[15] S. Gröblacher, T. Jennewein, A. Vaziri, G. Weihs, and A. Zeilinger, Experimental Quantum Cryptography with Qutrits, New J. Phys. 8, 75 (2006).

[16] J. T. Barreiro, T.-C. Wei, and P. G. Kwiat, Beating the Channel Capacity Limit for Linear Photonic Superdense Coding, Nat. Phys. 4, 282 (2008).

[17] J. Leach, E. Bolduc, D. J. Gauthier, and R. W. Boyd, Secure Information Capacity of Photons Entangled in Many Dimensions, Phys. Rev. A 85, 060304 (2012).

[18] U. M. Titulaer and R. J. Glauber, Density Operators for Coherent Fields, Phys. Rev. 145, 1041 (1966).

[19] J. Roslund, R. M. de Araújo, S. Jiang, C. Fabre, and N. Treps, Wavelength-Multiplexed Quantum Networks with Ultrafast Frequency Combs, Nat. Photonics 8, 109 (2013).

[20] V. A. Averchenko, V. Thiel, and N. Treps, Nonlinear Photon Subtraction from a Multimode Quantum Field, Phys. Rev. A 89, 063808 (2014).

[21] B. J. Smith and M. G. Raymer, Photon Wave Functions, Wave-Packet Quantization of Light, and Coherence Theory, New J. Phys. 9, 414 (2007).

[22] J. Schwinger, Unitary Operator Bases, Proc. Natl. Acad. Sci. U.S.A. 46, 570 (1960).

[23] C. H. Bennett and G. Brassard, in Proceedings of the IEEE International Conference on Computers, Systems and Signal Processing, Bangalore, India, 1984 (IEEE, New York, 1984), pp. 175-179; IBM Technical Disclosure Bulletin 28, 3153 (1985).

[24] D. T. Smithey, M. Beck, M. G. Raymer, and A. Faridani, Measurement of the Wigner Distribution and the Density Matrix of a Light Mode Using Optical Homodyne Tomography: Application to Squeezed States and the Vacuum, Phys. Rev. Lett. 70, 1244 (1993).

[25] A. Eckstein, B. Brecht, and C. Silberhorn, A Quantum Pulse Gate Based on Spectrally Engineered Sum Frequency Generation, Opt. Express 19, 13770 (2011).

[26] B. Brecht, A. Eckstein, R. Ricken, V. Quiring, H. Suche, L. Sansoni, and C. Silberhorn, Demonstration of Coherent Time-Frequency Schmidt Mode Selection Using DispersionEngineered Frequency Conversion, Phys. Rev. A 90, 030302(R) (2014).

[27] D. V. Reddy, M. G. Raymer, and C. J. McKinstrie, Efficient Sorting of Quantum-Optical Wave Packets by TemporalMode Interferometry, Opt. Lett. 39, 2924 (2014).

[28] D. V. Reddy, M. G. Raymer, and C. J. McKinstrie, Sorting Photon Wave Packets Using Temporal-Mode Interferometry Based on Multiple-Stage Quantum Frequency Conversion, Phys. Rev. A 91, 012323 (2015).

[29] C. K. Law, I. A. Walmsley, and J.H. Eberly, Continuous Frequency Entanglement: Effective Finite Hilbert Space and Entropy Control, Phys. Rev. Lett. 84, 5304 (2000). 
[30] W. P. Grice and I. A. Walmsley, Spectral Information and Distinguishability in Type-II Down-Conversion with a Broadband Pump, Phys. Rev. A 56, 1627 (1997).

[31] T. E. Keller and M.H. Rubin, Theory of Two-Photon Entanglement for Spontaneous Parametric DownConversion Driven by a Narrow Pump Pulse, Phys. Rev. A 56, 1534 (1997).

[32] A. B. U'Ren, C. Silberhorn, K. Banaszek, I. A. Walmsley, R. Erdmann, W. P. Grice, and M. G. Raymer, Generation of Pure-State Single-Photon Wavepackets by Conditional Preparation Based on Spontaneous Parametric Downconversion, Laser Phys. 15, 146 (2005).

[33] P. J. Mosley, J. S. Lundeen, B. J. Smith, P. Wasylczyk, A. B. U'Ren, C. Silberhorn, and I. A. Walmsley, Heralded Generation of Ultrafast Single Photons in Pure Quantum States, Phys. Rev. Lett. 100, 133601 (2008).

[34] O. Kuzucu, F. N. C. Wong, S. Kurimura, and S. Tovstonog, Joint Temporal Density Measurements for Two-Photon State Characterization, Phys. Rev. Lett. 101, 153602 (2008).

[35] X. Shi, A. Valencia, M. Hendrych, and J. P. Torres, Generation of Indistinguishable and Pure Heralded Single Photons with Tunable Bandwidth, Opt. Lett. 33, 875 (2008).

[36] A. Eckstein, A. Christ, P. J. Mosley, and C. Silberhorn, Highly Efficient Single-Pass Source of Pulsed Single-Mode Twin Beams of Light, Phys. Rev. Lett. 106, 013603 (2011).

[37] G. Harder, V. Ansari, B. Brecht, T. Dirmeier, C. Marquardt, and C. Silberhorn, An Optimized Photon Pair Source for Quantum Circuits, Opt. Express 21, 13975 (2013).

[38] B. Brecht, A. Eckstein, A. Christ, H. Suche, and C. Silberhorn, From Quantum Pulse Gate to Quantum Pulse Shaper-Engineered Frequency Conversion in Nonlinear Optical Waveguides, New J. Phys. 13, 065029 (2011).

[39] D. Méchin, R. Provo, J. D. Harvey, and C. J. McKinstrie, 180-nm Wavelength Conversion Based on Bragg Scattering in an Optical Fiber, Opt. Express 14, 8995 (2006).

[40] H. J. McGuinness, M. G. Raymer, C. J. McKinstrie, and S. Radic, Wavelength Translation Across $210 \mathrm{~nm}$ in the Visible Using Vector Bragg Scattering in a Birefringent Photonic Crystal Fiber, IEEE Photonics Technol. Lett. 23, 109 (2011).

[41] H. J. McGuinness, M. G. Raymer, C. J. McKinstrie, and S. Radic, Quantum Frequency Translation of Single-Photon States in a Photonic Crystal Fiber, Phys. Rev. Lett. 105, 093604 (2010).

[42] M. G. Raymer, S. J. van Enk, C. J. McKinstrie, and H. J. McGuinness, Interference of Two Photons of Different Color, Opt. Commun. 283, 747 (2010).

[43] D. V. Reddy, M. G. Raymer, C. J. McKinstrie, L. Mejling, and K. Rottwitt, Temporal Mode Selectivity by Frequency Conversion in Second-Order Nonlinear Optical Waveguides, Opt. Express 21, 13840 (2013).

[44] A. Christ, B. Brecht, W. Mauerer, and C. Silberhorn, Theory of Quantum Frequency Conversion and Type-II Parametric Down-Conversion in the High-Gain Regime, New J. Phys. 15, 053038 (2013).

[45] N. Quesada and J. E. Sipe, Effects of Time Ordering in Quantum Nonlinear Optics, Phys. Rev. A 90, 063840 (2014).
[46] Y.-P. Huang and P. Kumar, Mode-Resolved Photon Counting via Cascaded Quantum Frequency Conversion, Opt. Lett. 38, 468 (2013).

[47] A. S. Kowligy, P. Manurkar, N. V. Corzo, V. G. Velev, M. Silver, R. P. Scott, S. J. B. Yoo, P. Kumar, G. S. Kanter, and Y.-P. Huang, Quantum Optical Arbitrary Waveform Manipulation and Measurement in Real Time, Opt. Express 22, 27942 (2014).

[48] J. M. Donohue, M. D. Mazurek, and K. J. Resch, Theory of High-Efficiency Sum-Frequency Generation for SinglePhoton Waveform Conversion, Phys. Rev. A 91, 033809 (2015).

[49] A. Monmayrant, S. Weber, and B. Chatel, A Newcomer's Guide to Ultrashort Pulse Shaping and Characterization, J. Phys. B 43, 103001 (2010).

[50] H. de Riedmatten, I. Marcikic, W. Tittel, H. Zbinden, and N. Gisin, Quantum Interference with Photon Pairs Created in Spatially Separated Sources, Phys. Rev. A 67, 022301 (2003).

[51] R. Kaltenbaek, B. Blauensteiner, M. Żukowski, M. Aspelmeyer, and A. Zeilinger, Experimental Interference of Independent Photons, Phys. Rev. Lett. 96, 240502 (2006).

[52] J. Eisert, S. Scheel, and M. B. Plenio, Distilling Gaussian States with Gaussian Operations is Impossible, Phys. Rev. Lett. 89, 137903 (2002).

[53] A. M. Brańczyk, A. Fedrizzi, T. M. Stace, T. C. Ralph, and A. G. White, Engineered Optical Nonlinearity for a Quantum Light Source, Opt. Express 19, 55 (2011).

[54] P. Ben Dixon, J. H. Shapiro, and F. N. C. Wong, Spectral Engineering by Gaussian Phase-Matching for Quantum Photonics, Opt. Express 21, 5879 (2013).

[55] A. Dosseva, L. Cincio, and A. M. Branczyk, Shaping the Spectrum of Downconverted Photons through Optimized Custom Poling, arXiv:1410.7714.

[56] J. Nunn, L. J. Wright, C. Söller, L. Zhang, I. A. Walmsley, and B. J. Smith, Large-Alphabet Time-Frequency Entangled Quantum Key Distribution by Means of Time-to-Frequency Conversion, Opt. Express 21, 15959 (2013).

[57] P. C. Humphreys, W. Steven Kolthammer, J. Nunn, M. Barbieri, A. Datta, and I. A. Walmsley, ContinuousVariable Quantum Computing in Optical Time-Frequency Modes Using Quantum Memories, Phys. Rev. Lett. 113, 130502 (2014).

[58] M. Reck, A. Zeilinger, H. J. Bernstein, and P. Bertani, Experimental Realization of Any Discrete Unitary Operator, Phys. Rev. Lett. 73, 58 (1994).

[59] G. Gilbert, M. Hamrick, and Y.S. Weinstein, Efficient Construction of Photonic Quantum-Computational Clusters, Phys. Rev. A 73, 064303 (2006).

[60] D. E. Browne and T. Rudolph, Resource-Efficient Linear Optical Quantum Computation, Phys. Rev. Lett. 95, 010501 (2005).

[61] E. Knill, R. Laflamme, and G. J. Milburn, A Scheme for Efficient Quantum Computation with Linear Optics, Nature (London) 409, 46 (2001).

[62] R. Raussendorf and H.J. Briegel, A One-Way Quantum Computer, Phys. Rev. Lett. 86, 5188 (2001).

[63] K. F. Reim, P. Michelberger, K. C. Lee, J. Nunn, N. K. Langford, and I. A. Walmsley, Single-Photon-Level 
Quantum Memory at Room Temperature, Phys. Rev. Lett. 107, 053603 (2011).

[64] J. Nunn, I. A. Walmsley, M. G. Raymer, K. Surmacz, F. C. Waldermann, Z. Wang, and D. Jaksch, Mapping Broadband Single-Photon Wave Packets into an Atomic Memory, Phys. Rev. A 75, 011401 (2007).

[65] Z. Zheng, O. Mishina, N. Treps, and C. Fabre, Atomic Quantum Memory for Multimode Frequency Combs, Phys. Rev. A 91, 031802 (2015).
[66] R. C. Alferness, V. R. Ramaswamy, S. K. Korotky, M. D. Divino, and L.L. Buhl, Efficient Single-Mode Fiber to Titanium Diffused Lithium-Niobate Waveguide Coupling for $\gamma=1.32 \mu \mathrm{m}$, IEEE J. Quantum Electron. 18, 1807 (1982).

[67] K.-H. Luo, H. Herrmann, S. Krapick, B. Brecht, R. Ricken, V. Quiring, H. Suche, W. Sohler, and C. Silberhorn, Direct Generation of Genuine Single-Longitudinal-Mode Narrowband Photon Pairs, arXiv:1504.01854. 